\title{
Fujita Exponent for a Nonlinear Degenerate Parabolic Equation with Localized Source
}

\author{
Yulan Wang, ${ }^{1}$ Xiaojun Song, ${ }^{2}$ and Chao $\mathrm{Ye}^{3}$ \\ ${ }^{1}$ School of Mathematics and Computer Engineering, Xihua University, Chengdu 610039, China \\ ${ }^{2}$ College of Mathematic and Information, China West Normal University, Nanchong 637002, China \\ ${ }^{3}$ Division of Academic Periodicals, Xihua University, Chengdu 610039, China
}

Correspondence should be addressed to Yulan Wang; wangyulan-math@163.com

Received 27 April 2014; Accepted 10 July 2014; Published 24 August 2014

Academic Editor: Hagen Neidhardt

Copyright (C) 2014 Yulan Wang et al. This is an open access article distributed under the Creative Commons Attribution License, which permits unrestricted use, distribution, and reproduction in any medium, provided the original work is properly cited.

This paper is devoted to understand the blow-up properties of reaction-diffusion equations which combine a localized reaction term with nonlinear diffusion. In particular, we study the critical exponent of a $p$-Laplacian equation with a localized reaction. We obtain the Fujita exponent $q_{c}$ of the equation.

\section{Introduction}

This short paper is devoted to the Fujita critical exponent of the following $p$-Laplacian equation:

$$
\begin{gathered}
u_{t}=\left(\left|u_{x}\right|^{p-2} u_{x}\right)_{x}+a(x) u^{q}, \quad x \in \mathbb{R}, t>0, \\
u(x, 0)=u_{0}(x), \quad x \in \mathbb{R},
\end{gathered}
$$

where $p>2, q>0$, and the initial data $u_{0}(x)$ is nonnegative continuous and compactly supported.

We take the coefficient $a(x) \geq 0$ which is a compactly supported function, which means that the reaction term acts only locally. Thus, the model may be used to describe a chemical reaction-diffusion process in which, due to the effect of the catalyst, the reaction takes place only at some local sites [1].

As a representative example of quasilinear reactiondiffusion equation, classical $p$-Laplacian equation with no source term $(a(x) \equiv 0$ in (1)) and its variants had been extensively studied in the past few decades (see [2-6] and references therein). It is well known that $p$-Laplacian equation in general does not allow for classical solvability due to the nonlinearity and degeneracy. Therefore, the "solution" to a $p$ Laplacian means the weak solution in the usual integral sense (see, e.g., [4]). Among other things, previous studies show that blow-up happens for the problem with homogeneous reaction; that is, $a(x) \equiv 1$ :

$$
\begin{gathered}
u_{t}=\left(\left|u_{x}\right|^{p-2} u_{x}\right)_{x}+u^{q}, \quad x \in \mathbb{R}, t>0, \\
u(x, 0)=u_{0}(x), \quad x \in \mathbb{R} .
\end{gathered}
$$

It is shown that if $0<q \leq 1$, every solution is global in time, if $1<q \leq 2 p-1$, all solutions blow up, and if $q>2 p-1$, both global in time solutions and blowing up solutions exist. In this case, the numbers $q_{0}=1$ and $q_{c}=2 p-1$ are called the global existence exponent and the Fujita exponent, respectively. On the other hand, we remark that there is a close connection of problem (1) with the problem of diffusion with flux conditions on the boundary:

$$
\begin{gathered}
u_{t}=\left(\left|u_{x}\right|^{p-2} u_{x}\right)_{x}, \quad x>0, t>0, \\
-\left|u_{x}\right|^{p-2} u_{x}=u^{q}, \quad x=0, t \geq 0, \\
u(x, 0)=u_{0}(x), \quad x>0 .
\end{gathered}
$$

In fact, if we take a sequence of reaction coefficients converging to a Dirac delta at the origin (i.e., if $a_{n}(x) \rightarrow \delta_{0}(x)$ ), the corresponding solutions $u_{n}$ of (1) should converge to a solution of problem (3). In [7], authors proved that the global existence exponent of (3) is $q_{0}=2(p-1) / p$ and the Fujita 
exponent is $q_{c}=2(p-1)$. After that, there are plenty of works that extended their study to doubly degenerate equation or fast diffusion case (see, e.g., [8-10] and references therein).

The motivation of this short paper is the wish to understand the blow-up properties of reaction-diffusion equations which combine a nonlinear diffusion and localized reaction term, and this is the main difference with the existing studies of blow-up for similar reaction-diffusion equations. For the porous medium equation case $u_{t}=\left(u^{m}\right)_{x x}+a(x) u^{q}$, Ferreira et al. [11] proved that $q_{0}=(m+1) / 2, q_{c}=m+1$.

In our previous work, we has obtained the global existence exponent of (1) is $q_{0}=2(p-1) / p$. In this note, we will prove that the Fujita exponent of (1) is $q_{c}=2(p-1)$. Thus, it is the same as that of (3), and this is coincident with our intuitive judgement. We will modify several techniques developed in [11] to prove our main result.

\section{Some Preliminary Lemmas}

In this section, we will give some preliminary lemmas, whose proofs may be independent and interesting. We first have the following lemma from [3].

Lemma 1. There exists a positive constant $L_{s}<\infty$ such that problem

$$
\begin{gathered}
\left(\left|w^{\prime}\right|^{p-2} w^{\prime}\right)^{\prime}-\frac{1}{p-2} w+w^{p-1}=0, \quad 0<x<L_{s} \\
w^{\prime}(0)=0 \\
w^{\prime}\left(L_{s}\right)=w\left(L_{s}\right)=0
\end{gathered}
$$

has a unique nonnegative solution $w(x)$.

When $q>p-1$, we need to introduce the energy functional of (1) as follows:

$$
E(t)=\frac{1}{p} \int_{\mathbb{R}}\left|u_{x}\right|^{p} d x-\frac{1}{q+1} \int_{\mathbb{R}} a(x) u^{q+1} d x .
$$

Lemma 2. When $q>p-1$, if there exists $t_{0}>0$ such that $E\left(t_{0}\right)<0$, then all solutions to problem (1) blow up in finite time.

Proof. It is easy to verify that

$$
\begin{aligned}
E^{\prime}(t) & =\int_{\mathbb{R}} u_{x t}\left(\left|u_{x}\right|^{p-2} u_{x}\right) d x-\int_{\mathbb{R}} a(x) u^{q} u_{t} d x \\
& =-\int_{\mathbb{R}}\left|u_{t}\right|^{2} \leq 0,
\end{aligned}
$$

which shows that $E(t)$ is decreasing. Thus, $E(t) \leq E\left(t_{0}\right)<0$ for any $t \geq t_{0}$.

Define

$$
M(t)=\frac{1}{2} \int_{0}^{t} \int_{\mathbb{R}} u^{2}(x, s) d x d s
$$

For any $t \geq t_{0}$, we have $M(t)>0$ and

$$
M^{\prime}(t)=\frac{1}{2} \int_{\mathbb{R}} u^{2}(x, s) d x>0,
$$

$$
\begin{aligned}
M^{\prime \prime}(t)= & \int_{\mathbb{R}} u u_{t} d x \\
= & \int_{\mathbb{R}} u\left(\left(\left|u_{x}\right|^{p-2} u_{x}\right)_{x}+a(x) u^{q}\right) d x \\
= & -\int_{\mathbb{R}}\left|u_{x}\right|^{p} d x+\int_{\mathbb{R}} a(x) u^{q+1} d x \\
= & (q+1)\left(-\frac{1}{p} \int_{\mathbb{R}}\left|u_{x}\right|^{p} d x+\frac{1}{q+1} \int_{\mathbb{R}} a(x) u^{q+1} d x\right) \\
& -\left(1-\frac{q+1}{p}\right) \int_{\mathbb{R}}\left|u_{x}\right|^{p} d x .
\end{aligned}
$$

From $q>p-1$, it follows that $-(1-(q+1) / p)>0$. Thus,

$$
M^{\prime \prime}(t) \geq(q+1)(-E(t))>(q+1)\left(-E\left(t_{0}\right)\right)>0 .
$$

Namely, for any $t \geq t_{0}$, we have $M^{\prime \prime}(t)>c>0$ for some positive constant $c$.

Since $M^{\prime}\left(t_{0}\right)>0$ and $M\left(t_{0}\right)>0$, we obtain that $M^{\prime}(t) \geq$ $C t+C_{1}$, where $C_{1}$ is a constant. Therefore, $M^{\prime}(t) \rightarrow \infty$ as $t \rightarrow \infty$.

Next, we will prove that there exists $T<\infty$ such that $M(t) \rightarrow \infty$ as $t \rightarrow T$.

It follows from $E^{\prime}(t)=\int_{\mathbb{R}}\left|u_{t}\right|^{2} d x$ that

$$
E(t)-E\left(t_{0}\right)=\int_{t_{0}}^{t} E^{\prime}(s) d s=-\int_{t_{0}}^{t} \int_{\mathbb{R}}\left|u_{s}\right|^{2} d x d s .
$$

Since $E\left(t_{0}\right)<0$, we have $E(t)<-\int_{t_{0}}^{t} \int_{\mathbb{R}}\left|u_{s}\right|^{2} d x d s$. Thus, by (9),

$$
\begin{aligned}
M^{\prime \prime}(t) & =(q+1)(-E(t))-\left(1-\frac{q+1}{p}\right) \int_{\mathbb{R}}\left|u_{x}\right|^{p} d x \\
& \geq(q+1) \int_{t_{0}}^{t} \int_{\mathbb{R}}\left|u_{s}\right|^{2} d x d s .
\end{aligned}
$$

Therefore, by using Hölder inequality, we could obtain

$$
\begin{aligned}
M M^{\prime \prime}(t) & \geq \frac{q+1}{2}\left(\int_{t_{0}}^{t} \int_{\mathbb{R}}\left|u_{s}\right|^{2} d x d s\right)\left(\int_{t_{0}}^{t} \int_{\mathbb{R}} u^{2} d x d s\right) \\
& >\frac{q+1}{2}\left(\int_{t_{0}}^{t} \int_{\mathbb{R}} u u_{s} d x d s\right)^{2} \\
& =\frac{q+1}{2}\left(\frac{1}{2} \int_{\mathbb{R}} u^{2}(x, t) d x-\frac{1}{2} \int_{\mathbb{R}} u^{2}\left(x, t_{0}\right) d x\right) \\
& =\frac{q+1}{2}\left(M^{\prime}(t)-M^{\prime}\left(t_{0}\right)\right)^{2} .
\end{aligned}
$$


Due to the fact that $M^{\prime}(t) \rightarrow \infty$ as $t \rightarrow \infty$ and since $(q+$ 1) $/ 2>1$, there exists a constant $\alpha>0$ such that

$$
M(t) M^{\prime \prime}(t) \geq(1+\alpha)\left(M^{\prime}(t)\right)^{2},
$$

when $t$ is large enough. This is equivalent to the proposition that $M^{-\alpha}$ is a concave function.

Since $M(t) \geq 0$ and $M(t)$ is increasing, there exists $0<$ $T<\infty$ such that $M^{-\alpha}(T)=0$. Therefore, $M(t) \rightarrow \infty$ as $t \rightarrow T$.

Furthermore, we claim that $M^{\prime \prime}(t) \rightarrow \infty$ as $t \rightarrow T$. Otherwise, $M^{\prime \prime}(t)$ is bounded in $[0, T]$. Assume that $M^{\prime \prime}(t) \leq$ $2 C_{2}$ for $t \in[0, T]$, where $C_{2}$ is a positive constant.

Integrating the inequality twice over the interval $[0, T]$, we obtain that

$$
M(t) \leq C_{2} t^{2}+C_{3} t+C_{4}, \quad t \in[0, T] .
$$

The right side is bounded in $[0, T]$, and so is $M(t)$, which contradicts the fact that $M(t) \rightarrow \infty$ as $t \rightarrow T$. Hence,

$$
M^{\prime \prime}(t) \longrightarrow \infty
$$

in the limit $t \rightarrow T$.

Next, we prove that $u(x, t)$ blows up in finite time. By (1),

$$
\begin{aligned}
M^{\prime \prime}(t) & =-\int_{\mathbb{R}}\left|u_{x}\right|^{p} d x+\int_{\mathbb{R}} a(x) u^{q+1} d x \leq \int_{\mathbb{R}} a(x) u^{q+1} d x \\
& \leq \operatorname{meas}(\operatorname{supp} a)\|u(\cdot, t)\|_{\infty}^{q+1} .
\end{aligned}
$$

Therefore, $u(x, t)$ blows up in the sense of $L^{\infty}$ norm from (16).

Lemma 3. If $q>2(p-1)$ and $\sigma<-1$, Cauchy problem

$$
\begin{gathered}
u_{t}=\left(\left|u_{x}\right|^{p-2} u_{x}\right)_{x}+|x|^{\sigma} u^{q}, \\
u(x, 0)=u_{0}(x)
\end{gathered}
$$

has a global supersolution.

Proof. Consider the following Cauchy problem:

$$
\begin{gathered}
u_{t}=\left(\left|u_{x}\right|^{p-2} u_{x}\right)_{x}, \quad x \in \mathbb{R}, t>0, \\
u(x, 0)=L|x|^{-1}, \quad x \in \mathbb{R} .
\end{gathered}
$$

The existence and uniqueness of the solution to this problem was established in $[5,6,12]$. The solution has the form

$$
U(x, t)=t^{-\beta} g_{L}\left(|x| t^{-\beta}\right), \quad \beta=\frac{1}{2(p-1)},
$$

where $g_{L}$ is a positive, bounded, and decreasing function and satisfies the following boundary value problem:

$$
\begin{gathered}
\left(\left|g_{L}^{\prime}\right|^{p-2} g_{L}^{\prime}\right)^{\prime}+\beta r g_{L}^{\prime}+\beta g_{L}=0, \quad r>0, \\
g_{L}^{\prime}(0)=0, \quad \lim _{r \rightarrow \infty} r g_{L}(r)=L .
\end{gathered}
$$

Set $z(x, t)=\lambda U\left(x, \lambda^{p-2} t+t_{0}\right)$, where $\lambda>0$ is to be determined later; $t_{0}$ is a constant between 0 and 1 .

One can see that $\lim _{r \rightarrow \infty} r g_{L}(r)=L$ and $\sigma<-1$ show that $\lim _{r \rightarrow \infty} r^{\sigma /(q-1)} g_{L}(r)=0$. From (21), we know that

$$
\sup \left(|x|^{\sigma} \cdot\|z(x, t)\|_{\infty}\right) \leq C \lambda\left(\lambda^{p-2} t+t_{0}\right)^{-\beta(1-\sigma /(q-1))}
$$

for some $C>0$. Therefore, $z(x, t)$ is the solution of the following problem:

$$
\begin{gathered}
z_{t}=\left(\left|z_{x}\right|^{p-2} z_{x}\right)_{x}, \quad x \in \mathbb{R}, t>0, \\
z(x, 0)=\lambda U\left(x, t_{0}\right), \quad x \in \mathbb{R} .
\end{gathered}
$$

Next, we introduce the function $v(x, t)=A(t) z(x, B(t))$, where $A(t)$ and $B(t)$ are solutions of the following ordinary differential system:

$$
\begin{gathered}
A^{\prime}(t)=C^{q-1} \lambda^{q-1}\left(\lambda^{p-2} B(t)+t_{0}\right)^{-\beta(q-1-\sigma)} A^{q}(t), \quad t>0, \\
B^{\prime}(t)=A^{p-2}(t), \quad t>0, \\
A(0)=1, \quad B(0)=0 .
\end{gathered}
$$

Then, it is easy to verify that $v(x, t)$ satisfies

$$
\begin{gathered}
v_{t} \geq\left(\left|v_{x}\right|^{p-2} v_{x}\right)_{x}+|x|^{\sigma} v^{q}, \quad x \in \mathbb{R}, t>0, \\
v(x, 0)=\lambda U\left(x, t_{0}\right), \quad x \in \mathbb{R} .
\end{gathered}
$$

Therefore, $v(x, t)$ is a supersolution of (18) if $u(x, 0) \leq$ $\lambda U\left(x, t_{0}\right)$. If $q>2(p-1)$, it is a global supersolution from the following Lemma 4 .

Lemma 4. If $q>2(p-1)$ and $\sigma<-1$, then there exists a positive constant $\lambda_{0}$ such that problem (25) has a global solution $(A(t), B(t))$ with $A(t)$ bounded in $[0,+\infty)$ if $0 \leq \lambda<$ $\lambda_{0}$.

Proof. The local existence and uniqueness of solution $(A(t), B(t))$ of (25) follows from the standard theory of initial value problem on ordinary differential equation. From (25), for $t>0$, we have $A^{\prime}(t)>0, A(t)>1$ and the solution continues as long as the solution exists and $A(t)$ is finite.

By (25), $B(t)$ is uniquely defined by $B(t)=\int_{0}^{t} A^{p-2}(s) d s$. Moreover,

$$
B(s)=\int_{0}^{s} A^{p-2}(s) d \tau \geq A^{p-2}(0) s=s
$$

for all $s \in[0, t]$. From $\sigma<-1$ and $q>2(p-1)$, we have $\beta(q-1-\sigma)-1>0$. So

$$
\begin{aligned}
1-A^{1-q}(t) & =(q-1) C^{q-1} \lambda^{q-1} \int_{0}^{t}\left(\lambda^{p-2} B(s)+t_{0}\right)^{-\beta(q-1-\sigma)} d s \\
& \leq \frac{q-1}{\beta(q-1-\sigma)-1} C^{q-1} \lambda^{q-1+p} t_{0}^{-\beta(q-1-\sigma)+1} .
\end{aligned}
$$


Let $\lambda=\lambda_{0}$ be a positive constant defined by

$$
\frac{q-1}{\beta(q-1-\sigma)-1} C^{q-1} \lambda^{q-1+p} t_{0}^{-\beta(q-1-\sigma)+1}=\frac{1}{2}
$$

and, given a fixed $\lambda \in\left(0, \lambda_{0}\right)$, by (28), we have $1 \leq A(t) \leq$ $2^{1 /(q-1)}$ as long as $A(t)$ exists globally. Meanwhile, it is easy to see that $t \leq B(t) \leq 2^{(p-2) /(q-1)}$ for all $t>0$; thus, $B(t)$ is global.

\section{Main Results and Their Proofs}

We first deal with the case of $q>2(p-1)$, which is easier than the other cases.

Theorem 5. If $q>2(p-1)$, then there exist both global solutions and blowing-up solutions to problem (1).

Proof. Lemma 3 states that when $q>2(p-1)$ and $\sigma<-1$, equation

$$
u_{t}=\left(\left|u_{x}\right|^{p-2} u_{x}\right)_{x}+|x|^{\sigma} u^{q}
$$

has a global supersolution.

Note that the reaction coefficient of this equation is bigger than $a(x)$, multiplied by a constant if necessary. So, we conclude that problem (1) has global solutions if $q>2(p-1)$ and $\sigma<-1$.

On the other hand, it is well known (see, e.g., [13]) that problem

$$
\begin{gathered}
u_{t}=\left(\left|u_{x}\right|^{p-2} u_{x}\right)_{x}+u^{q}, \quad x \in[-L, L], t>0, \\
u( \pm L, t)=0, \quad t>0, \\
u(x, 0)=u_{0}(x), \quad x \in[-L, L]
\end{gathered}
$$

admits a blowing-up solution, which is a subsolution to our problem for suitable $L$. We thus complete our proof.

Our remainder objective is to prove that if $2(p-1) / p<$ $q \leq 2(p-1)$, then all solutions blow up in finite time. And this result is composed of several theorems.

To simplify the exposition, we may take $a(x)$, a characteristic function; $a(x)=\chi_{[-L, L]}$ for some $0<L<+\infty$ in the remainder of this paper. Note that such assumption imposing on $a(x)$ is not essential since it can be modified to a more general compactly function.

Theorem 6. If $2(p-1) / p<q<p-1$, then all the solutions to problem (1) blow up in finite time.

Proof. We will prove this result by the similar idea of Lemma 10 in [11]. We firstly construct here a blow-up subsolution matching of a self-similar function with a blowing-up parabola.

Fix a point $0<x_{0} \leq L$ and consider the even function obtained by the reflection of

$$
\underline{u}(x, t)= \begin{cases}\left(A(t)-B(t) x^{p /(p-1)}\right)_{+}, & 0 \leq x \leq x_{0}, \\ V\left(x-x_{0}, t\right), & x \geq x_{0},\end{cases}
$$

where $A(t)$ and $B(t)$ are to be determined later and $V$ is a selfsimilar solution of the problem

$$
\begin{aligned}
& V_{t}=\left(\left|V_{x}\right|^{p-2} V_{x}\right)_{x}, \quad x>0, t>0, \\
& -\left|V_{x}\right|^{p-2} V_{x}=V^{s}, \quad x=0, t>0 .
\end{aligned}
$$

It is known from [7] that problem (33) admits blowing-up self-similar solution if $s>2(p-1) / p$, and they have the form

$$
V(x, t)=(T-t)^{-\gamma} \Theta\left(x(T-t)^{(s-(p-1)) /(p-1) \cdot \gamma}\right),
$$

where $\gamma=(p-1) /(s \cdot p-2(p-1))$.

In order to have a $C^{1}$ function $\underline{u}(x, t)$, we need

$$
\begin{gathered}
A(t)-B(t) x_{0}^{p /(p-1)}=V(0, t), \\
-\frac{p}{p-1} B(t) x_{0}^{1 /(p-1)}=V^{\prime}(0) .
\end{gathered}
$$

To this end, we take

$$
\begin{gathered}
A(t)=V(0, t)+\frac{p-1}{p} x_{0} V^{s /(p-1)}(0, t), \\
B(t)=\frac{p-1}{p} x_{0}^{-1 /(p-1)} V^{s /(p-1)}(0, t) .
\end{gathered}
$$

Henceforth, for $0 \leq x \leq x_{0}$, the function $\underline{u}(x, t)$ has the following form:

$$
\begin{gathered}
\underline{u}(x, t)=(T-t)^{-\gamma} I(x, t), \\
I(x, t)=\Theta(0)+\frac{p-1}{p} x_{0}^{1 /(1-p)}\left(x_{0}^{p /(p-1)}-x^{p /(p-1)}\right) \\
\times \Theta^{s /(p-1)}(0)(T-t)^{\gamma(1-s /(p-1))} .
\end{gathered}
$$

Notice that if $s \leq p-1, I(x, t) \sim$ const. In order to see that $\underline{u}$ is a subsolution to problem (1), we only have to look at the interval $\left(0, x_{0}\right)$. Direct calculations show

$$
\begin{aligned}
& \underline{u}_{t}=(T-t)^{-\gamma-1}(\gamma I-\frac{\gamma(p-1-s)}{p} \\
& \times x_{0}^{1 /(1-p)}\left(x_{0}^{p /(p-1)}-x^{p /(p-1)}\right) \\
& \times\left.\Theta^{s /(p-1)}(0)(T-t)^{\gamma(1-s /(p-1))}\right), \\
&\left(\left|\underline{u}_{x}\right|^{p-2} \underline{u}_{x}\right)_{x}=-\frac{\Theta^{s}(0)}{x_{0}}(T-t)^{-\gamma s}, \\
& \underline{u}^{q}=(T-t)^{-q \gamma} I^{q} .
\end{aligned}
$$

Therefore, when $T$ is small, $\underline{u}$ is a subsolution to problem (1) if the inequality

$$
C_{1} T^{-\gamma-1} \leq-C_{2} T^{-\gamma s}+C_{3} T^{-q \gamma}
$$

is valid. 
This inequality could be achieved if we take $s<$ $\min \{q,(p-1)(q+1) / p\}$. Since $2(p-1) / p<q<(p-1)$, $q<(p-1)(q+1) / p$. So, we only need to choose $2(p-1) / p<$ $s<q$ to get the desired blow-up subsolution.

Now, we claim that all solutions to (1) blow up in finite time. Suppose on the contrary that $u(x, t)$ exist globally. From Lemma 6 of [11], we know that there exists some $t_{0}>0$ such that $u\left(x, t_{0}\right) \geq \underline{u}(x, 0)$. Henceforth, $u\left(x, t_{0}+t\right) \geq \underline{u}(x, t)$ by comparison theorem. And this implies that $u(x, t)$ blows up. This contradicts the hypothesis that $u(x, t)$ exist globally.

Theorem 7. If $q=p-1$, then every solution to problem (1) blows up in finite time.

Proof. We first prove that problem (1) admits, for any length $L>0$, a unique symmetric blow-up solution in the selfsimilar form

$$
U(x)=(T-t)^{-1 /(p-2)} F(x) .
$$

Substituting $U$ into the first equation of (1), we have that the profile $F$ satisfies the equation

$$
\left(\left|F^{\prime}\right|^{p-2} F^{\prime}\right)^{\prime}-\frac{1}{p-2} F+a(x) F^{p-1}=0, \quad x>0
$$

plus the boundary condition $F^{\prime}(0)=0$.

If $L \geq L_{s}$, that is, $\left[-L_{s}, L_{s}\right] \subset \operatorname{supp} a(x),(T-t)^{-1 /(p-2)} w(x)$ is the desired blow-up solution, where $w(x)$ is defined in Lemma 1.

If $L<L_{s}$, we construct $F$ by putting together two pieces as in [11]. For $x \geq L$, since $a(x)=0$, it is easy to verify that

$$
F(x)=C\left(L_{0}-x\right)_{+}^{p /(p-2)}
$$

satisfies the above equation for any $L_{0}>L$, where $C^{p-2}=$ $(1 / 2(p-1))((p-2) / p)^{p-1}$. If $0<x<L$, we consider the problem

$$
\begin{gathered}
\left(\left|\nu^{\prime}\right|^{p-2} v^{\prime}\right)^{\prime}-\frac{1}{p-2} \nu+v^{p-1}=0, \quad 0<x<L, \\
v(0)=A>\left(\frac{1}{p-2}\right)^{1 /(p-2)}, \quad \nu^{\prime}(0)=0, \\
v^{\prime}(L)=C\left(L_{0}-L\right)_{+}^{p /(p-2)} \\
v^{\prime}(L)=-\frac{p}{p-2} C\left(L_{0}-L\right)_{+}^{p /(p-2)} .
\end{gathered}
$$

Taking $m=1$ in Lemma 3.1 of [14], we know that, for every length $L<L_{s}$, there exists a positive solution $v$ to this problem. Therefore, $(T-t)^{-1 /(p-12)} v(x)$ is the desired selfsimilar blow-up solution.

We now check that the above self-similar solution constructed can be put below any solution if we let pass enough time. In fact, the self-similar solution has small initial value if $T>0$ is large, but its support is not small, since the length $L_{0}$ is not small. We then use the penetration property of the solutions to the $p$-Laplacian equation to guarantee that there exists $t_{0}>0$ such that the support of $u\left(\cdot, t_{0}\right)$ contains the interval $\left[-L_{0}, L_{0}\right]$. Therefore, we could get $u\left(x, t_{0}\right) \geq U(x, 0)$ by taking $T$ which is large enough.

By comparison, $u$ must blow up in finite time.

Now, we turn to the case of $q>p-1$.

Theorem 8. If $p-1<q<2(p-1)$, every solution to problem (1) blows up in finite time.

Proof. From Lemma 2, we know that if there exists $t_{0}>0$, such that energy functional $E_{u}\left(t_{0}\right)<0$, then all solutions to (1) blow up in finite time.

We now check that the so-called Barenblatt function

$$
\begin{aligned}
& u_{B}(x, t ; d) \\
& \quad=t^{-1 / 2(p-1)} D\left(d^{p /(p-1)}-x^{p /(p-1)} t^{-p / 2(p-1)^{2}}\right)_{+}^{(p-1) /(p-2)}
\end{aligned}
$$

satisfies the above requirement provided that $t$ is large. In fact,

$$
\begin{aligned}
& E_{u_{B}}(t)=\frac{1}{p}\left(\frac{D p}{p-2}\right)^{p} \\
& \times \int_{-\infty}^{+\infty} t^{-p(2 p-1) / 2(p-1)^{2}} \\
& \times\left(d^{p /(p-1)}-x^{p /(p-1)} t^{-p / 2(p-1)^{2}}\right)_{+}^{p /(p-2)} \\
& \times x^{p /(p-1)} d x \\
& -\frac{D}{q+1} \\
& \times \int_{-L}^{L} t^{-(q+1) / 2(p-1)} \\
& \times\left(d^{p /(p-1)}\right. \\
& \left.-x^{p /(p-1)} t^{-p / 2(p-1)^{2}}\right)_{+}^{(q+1)(p-1) /(p-2)} d x \\
& \leq C_{1}\left(\int_{d_{1} t^{1 / 2(p-1)}}^{d_{2} t^{1 / 2(p-1)}} x^{p /(p-1)} d x\right) \\
& \times t^{-p(2 p-1) / 2(p-1)^{2}}-C_{2} t^{(q+1) / 2(p-1)} \\
& \times\left(\int_{0}^{c L t^{-1 / 2(p-1)}}\left(1-z^{p /(p-1)}\right)_{+}^{(q+1)(p-1) /(p-2)} d x\right) \\
& \times t^{1 / 2(p-1)} \\
& \leq C_{1} t^{-\left(2 p^{2}-3 p+1\right) / 2(p-1)^{2}}-C_{3} t^{-(q+1) / 2(p-1)} .
\end{aligned}
$$


From $q<2(p-1)$, we know $-\left(2 p^{2}-3 p+1\right) / 2(p-1)^{2}<$ $-(q+1) / 2(p-1)$. Therefore, $E_{u_{B}(t)}<0$ provided that $t$ is large enough.

Finally, we may choose $d>0$ which is small in order to have $u_{B}(x, 1 ; d) \leq u_{0}(x)$. Therefore,

$$
u_{B}(x, t+1 ; d) \leq u(x, t) .
$$

Since the solution to problem (1) with initial value $u_{B}\left(\cdot, t_{1} ; d\right)$ with $t_{1}$ which is large blows up, so does $u$.

Now, we deal with the case of $q=2(p-1)$.

Theorem 9. If $q=2(p-1)$, every solution to (1) blows up in finite time.

Proof. Here, we argue as in [15] or [7]. The proof consists of several steps.

Step 1. Assume by contradiction that there exists a global nonnegative nontrival solutions $u(x, t)$ to problem (1). According to the symmetry properties of equation with $q=2(p-1)$, we introduce the following rescaled function:

$$
\begin{array}{r}
\theta(\xi, \tau)=(1+t)^{1 / 2(p-1)} u(x, t), \\
\xi=x(1+t)^{-1 / 2(p-1)}, \quad \tau=\ln (t+1) .
\end{array}
$$

Then, $\theta$ solves the equation

$$
\theta_{\tau}=\left(\left|\theta_{\xi}\right|^{p-2} \theta_{\xi}\right)_{\xi}+\frac{1}{2(p-1)}(\xi \theta)_{\xi}+\psi(\xi) e^{\tau / 2(p-1)} \theta^{2(p-1)},
$$

where $\psi(\xi)=\chi\left\{|\xi| \leq L e^{-\tau / 2(p-1)}\right\}$.

Denote by $g(\xi, \tau)$ the solution of $(48)$ with initial data

$$
g(\xi, 0)=D\left(d^{p /(p-1)}-\xi^{p /(p-1)}\right)_{+}^{(p-1) /(p-2)}=u_{B}(\xi, 1 ; d) .
$$

Assuming that without loss of generality $u_{0}(0)>0$, we have that there exists a small $d>0$ such that $u_{0}(x) \geq u_{B}(x, 1 ; d)$. This implies that $g(\xi, \tau) \leq \theta(\xi, \tau)$, and therefore $g$ is also a global solution.

Step 2. We now discuss some important properties of $g(\xi, \tau)$.

First, by applying the maximum principle to the linear parabolic equation for the derivative $z=g_{\xi}$ and by using a standard regularization argument, we have that $g(\xi, \tau)$ is nonincreasing in $\xi>0$. Next, for the special form of the initial data, it is easy to verify by the same method of $[7,15]$ that $g$ is increasing in $\tau$. Therefore, there exist

$$
f(\xi)=\lim _{\tau \rightarrow+\infty} g(\xi, \tau) .
$$

We claim that $f(\xi)<+\infty$.

In fact, this fails for some $\xi_{0}>0$ and hence $\lim _{\tau \rightarrow+\infty} g\left(\xi_{0}, \tau\right)=+\infty$. Since $g$ is nonincreasing in $\xi$, we conclude that given $M>0$ there exists $\tau_{M}$ which is large such that

$$
g\left(\xi, \tau_{M}\right)>M, \quad|\xi| \leq \xi_{0}
$$

Set $w(x, t)=e^{-\tau / 2(p-1)} g(\xi, \tau)$. We will show that by taking $M$ which is large enough $w$ blows up in finite time, which contradicts the fact that $g$ is global.

To this end we consider a special function $v(x)=$ $u_{B}(x, t ; d)$. Then $v(x)$ has negative energy, $\operatorname{supp}(v) \quad \subset$ $\left(-\xi_{0} e^{\tau_{M} / 2(p-1)}, \xi_{0} e^{\tau_{M} / 2(p-1)}\right)$ and $v(x) \leq M e^{-\tau_{M} / 2(p-1)}$. The first condition is achieved if $d$ is large enough. The last two requirements are possible for some $t=t_{*}$ provided that $M$ is chosen as large enough, depending on $\xi_{0}$ and $\tau_{M}$. This implies that the solution to (48) with initial value $v$ blows up in finite time. $w$ is larger than this solution, and this contradicts the fact that $g$ is global. The contradiction shows that $f(\xi)$ defined as above is finite for every $\xi \neq 0$.

Step 3. Using the monotonicity property, we can introduce a Lyapunov functional as in [15]. By means of Lyapunov functional, we could obtain as in [15] that $f$ is a weak symmetric solution to the equation

$$
\left(\left|\theta^{\prime}\right|^{p-2} \theta^{\prime}\right)^{\prime}+\frac{1}{2(p-1)}(\xi \theta)^{\prime}=0 .
$$

for every $\xi \neq 0$.

In order to finish the blow-up argument in this case, we observe that the only self-similar solution to this equation is (see [7])

$$
f(\xi)=D\left(d^{p /(p-1)}-\xi^{p /(p-1)}\right)_{+}^{(p-1) /(p-2)} .
$$

This is followed by uniqueness and a symmetrization argument. In particular, $f(0)$ is finite. Then, by passing to the limit in the weak formulation of (48), we could get the boundary condition at $\xi=0^{+}$as follows:

$$
\lim _{\xi \rightarrow 0^{+}}\left(\left|\theta^{\prime}\right|^{p-2} \theta^{\prime}\right)(\xi)=-2 L f^{2(p-1)}(0) .
$$

However, the profiles obtained do not satisfy this condition. This completes the proof in the critical case.

\section{Conclusion and Further Discussion}

Theorems 6-9 show that if $2(p-1) / p<q \leq 2(p-1)$, then all solutions to problem (1) blow up. On the other hand, from Theorem 5, we know both blow-up solutions and global solutions exist in the case of $q>2(p-1)$. Thus, the Fujita exponent of problem (1) is $q_{c}=2(p-1)$.

Comparing the proof of the main results in this paper and that of the corresponding part in [11], we find that we could deal with the doubly degenerate problem

$$
\begin{gathered}
u_{t}=\left(\left|\left(u^{m}\right)_{x}\right|^{p-2}\left(u^{m}\right)_{x}\right)_{x}+a(x) u^{q}, \quad x \in \mathbb{R}, t>0, \\
u(x, 0)=u_{0}(x), \quad x \in \mathbb{R},
\end{gathered}
$$

by similar a technique to the present paper. One can conjecture that the Fujita exponent of this Cauchy problem is $q=(m+1)(p-1)$. 


\section{Disclosure}

After this paper was submitted, the authors found that, in [16], Liang studied the model (1) in general Ndimensional setting. The Fujita exponent obtained in this paper is consistent with that of [16] in the case of $N=1$. However, we have some new idea and different proofs here due to the special 1-dimensional setting. Besides the Fujita exponent, we have obtained some by-products which are independent and interesting, such as Lemmas 2 and 3.

\section{Conflict of Interests}

The authors declare that there is no conflict of interests regarding the publication of this paper.

\section{Acknowledgments}

This work is supported by Scientific Research Found of Sichuan Provincial Education Department (12ZA288), Xihua University Young Scholars Training Program, and Applied Basic Research Project of Sichuan Province (2013JY0178).

\section{References}

[1] K. Bimpong-Bota, P. Ortoleva, and J. Ross, "Far-fromequilibrium phenomena at local sites of reaction," The Journal of Chemical Physics, vol. 60, no. 8, pp. 3124-3133, 1974.

[2] E. DiBenedetto, Degenerate Parabolic Equations, Springer, New York, NY, USA, 1993.

[3] Z. L. Liang and J. N. Zhao, "Localization for the evolution p-Laplacian equation with strongly nonlinear source term," Journal of Differential Equations, vol. 246, no. 1, pp. 391-407, 2009.

[4] Z. Q. Wu, J. N. Zhao, J. X. Yin, and H. Li, Nonlinear Diffusion Equations, World Scientific Publishing, River Edge, NJ, USA, 2001.

[5] J. N. Zhao, "The asymptotic behaviour of solutions of a quasilinear degenerate parabolic equation," Journal of Differential Equations, vol. 102, no. 1, pp. 33-52, 1993.

[6] J. N. Zhao, "On the Cauchy problem and initial traces for the evolution $p$-Laplacian equations with strongly nonlinear sources," Journal of Differential Equations, vol. 121, no. 2, pp. 329-383, 1995.

[7] V. A. Galaktionov and H. A. Levine, "On critical Fujita exponents for heat equations with nonlinear flux conditions on the boundary," Israel Journal of Mathematics, vol. 94, pp. 125-146, 1996.

[8] Z. P. Li and C. L. Mu, "Critical curves for fast diffusive nonNewtonian equations coupled via nonlinear boundary flux," Journal of Mathematical Analysis and Applications, vol. 340, no. 2, pp. 876-883, 2008.

[9] Z. P. Li, C. L. Mu, and Z. J. Cui, "Critical curves for a fast diffusive polytropic filtration system coupled via nonlinear boundary flux," Zeitschrift für Angewandte Mathematik und Physik, vol. 60, no. 2, pp. 284-298, 2009.

[10] Z. J. Wang, J. X. Yin, and C. P. Wang, "Critical exponents of the non-Newtonian polytropic filtration equation with nonlinear boundary condition," Applied Mathematics Letters, vol. 20, no. 2, pp. 142-147, 2007.
[11] R. Ferreira, A. de Pablo, and J. L. Vazquez, "Classification of blow-up with nonlinear diffusion and localized reaction," Journal of Differential Equations, vol. 231, no. 1, pp. 195-211, 2006.

[12] E. DiBenedetto and M. A. Herrero, "On the Cauchy problem and initial traces for a degenerate parabolic equation," Transactions of the American Mathematical Society, vol. 314, no. 1, pp. 187-224, 1989.

[13] J. N. Zhao, "Existence and nonexistence of solutions for $u_{t}=$ $\operatorname{div}\left(|\nabla u|^{p-2} \nabla u\right)+f(\nabla u, u, x, t)$," Journal of Mathematical Anal$y$ sis and Applications, vol. 172, pp. 130-146, 1993.

[14] Z. Y. Xiang, "Localization for a doubly degenerate parabolic equation with strongly nonlinear sources," Mathematical Methods in the Applied Sciences, vol. 33, no. 9, pp. 1078-1088, 2010.

[15] V. A. Galaktionov, "Blow-up for quasilinear heat equations with critical Fujita's exponents," Proceedings of the Royal Society of Edinburgh, vol. 124, no. 3, pp. 517-525, 1994.

[16] Z. L. Liang, "Critical exponents for the evolution $p$-Laplacian equation with a localized reaction," Indian Journal of Pure and Applied Mathematics, vol. 43, no. 5, pp. 535-558, 2012. 


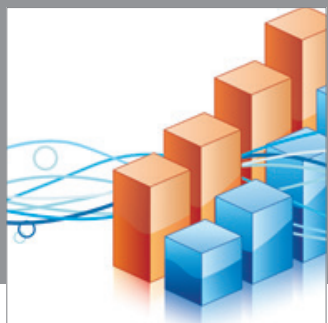

Advances in

Operations Research

mansans

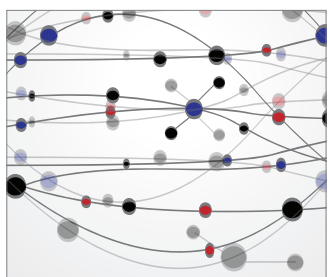

The Scientific World Journal
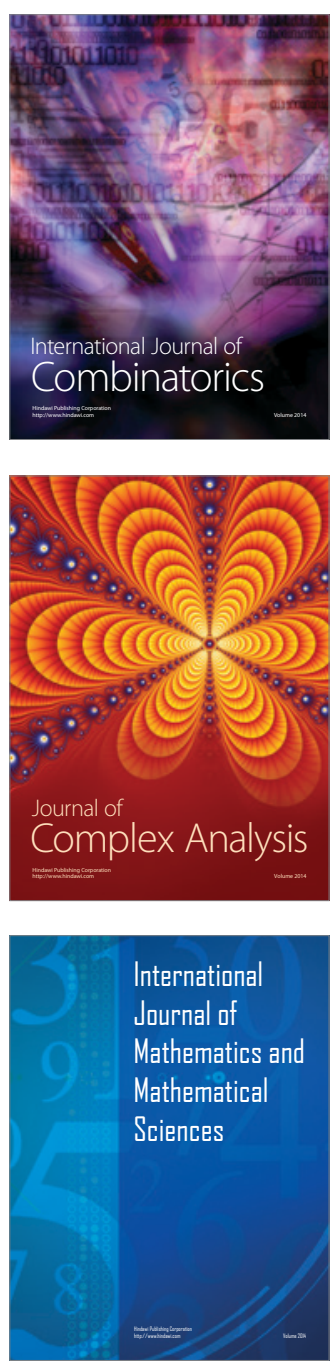
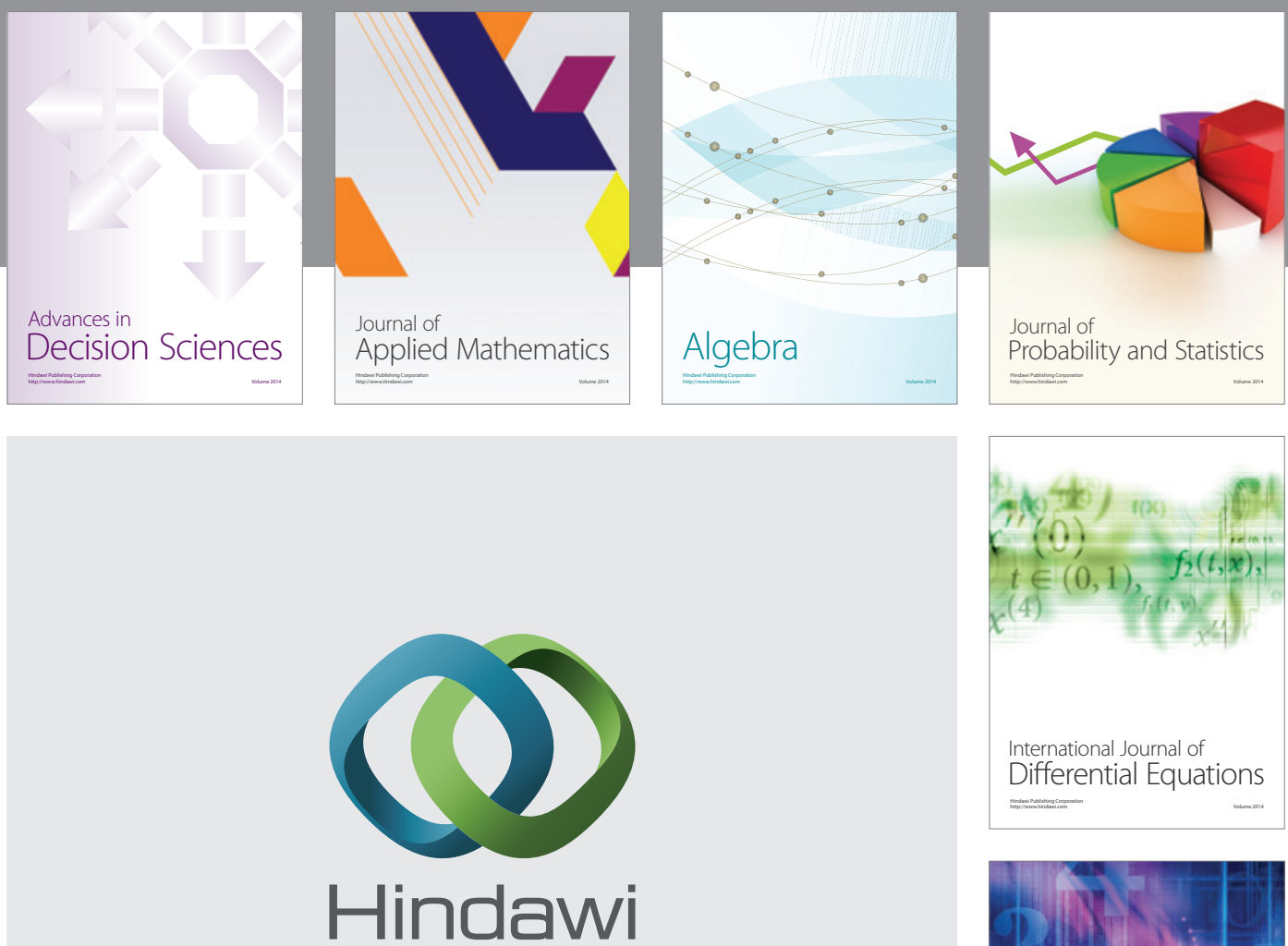

Submit your manuscripts at http://www.hindawi.com
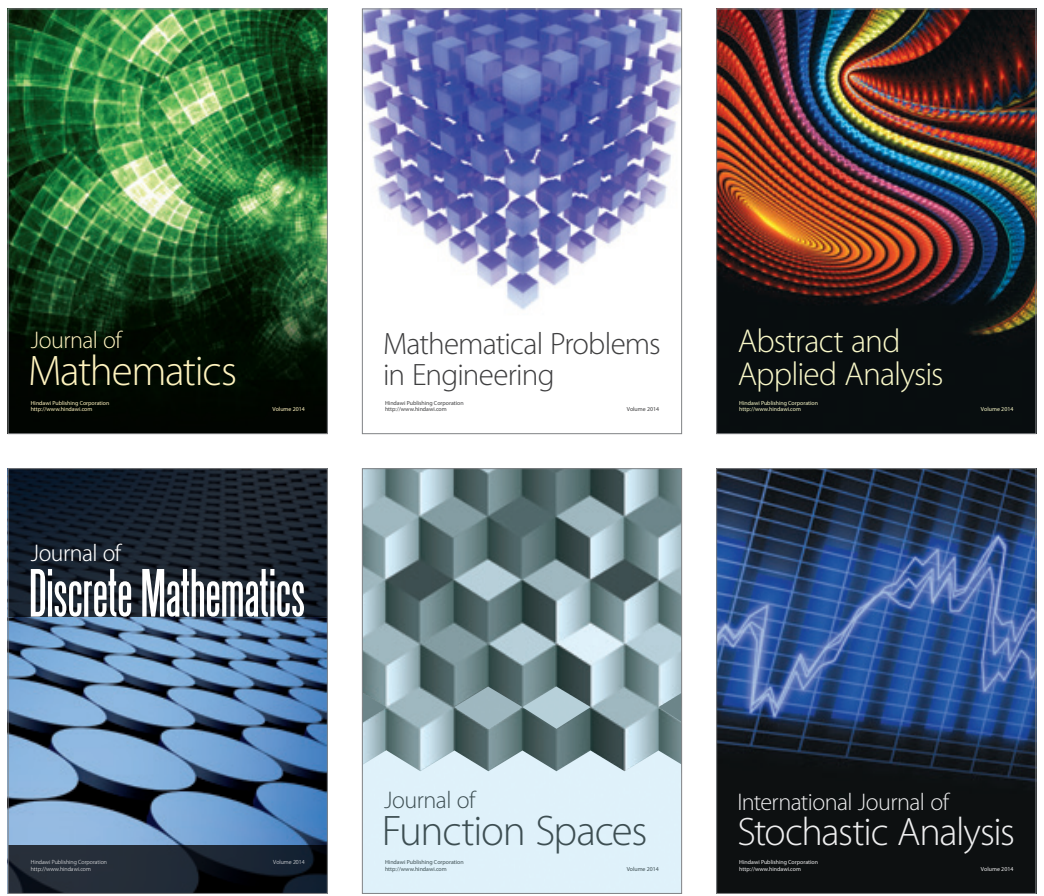

Journal of

Function Spaces

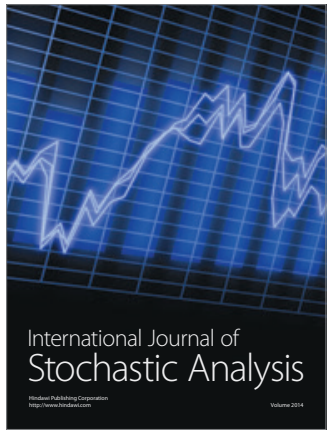

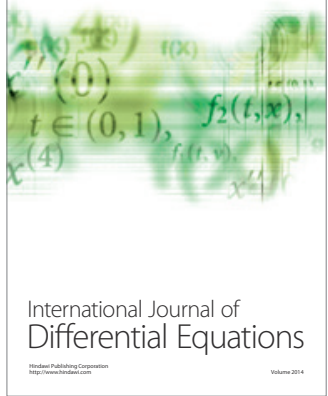
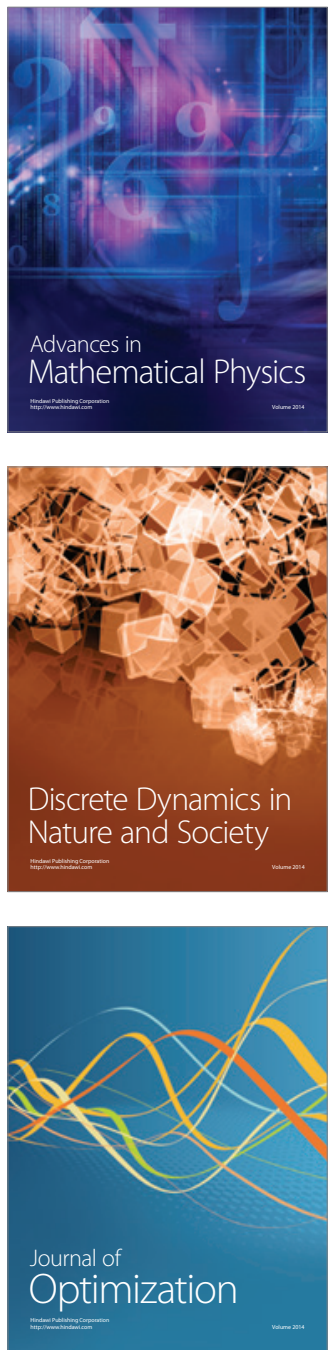